\title{
La implementación del concepto de desarrollo en las políticas de cooperación al desarrollo y en materia migratoria
}

\section{The concept of development in cooperation for de- velopment and migratory policies}

\author{
Nerea Azkona ${ }^{1}$
}

\section{Resumen:}

Es importante promover la sinergia entre migración y desarrollo, partiendo de un enfoque global que une migraciones y desarrollo para cumplir con la exigencia de una mayor coherencia de políticas, principio que pretende salvaguardar el resto de las políticas de interferencia en el objetivo propio de la cooperación. Sin embargo, también se ve la cooperación como una herramienta que puede contribuir a frenar los flujos migratorios. Este enfoque global ha fomentado el control de fronteras y la inclusión de la cooperación en las estrategias migratorias.

PAlabras Clave: desarrollo, coherencia, migración, cooperación internacional, condicionalidad de la AOD.

\section{Abstract:}

It is important to promote synergy between migration and development,

1 Consultora independiente de Azkorenas Consultoras: galmena@hotmail.com. Recibido el 31 de julio de 2013; aceptado el 6 de agosto de 2013. 
starting from a global approach joining migrations and development so as to meet the requirement of a greater coherence of policies, a principle aimed at safeguarding other policies from interfering in the purpose of cooperation. Nevertheless, cooperation is also seen as a tool that can help to stop migratory flows. This global approach has promoted the control of borders and the incorporation of cooperation in migration strategies.

KEYWORDS: development, coherence, migrations, international cooperation, conditionality of the ODA. 
Nerea Azkona • La implementación del concepto de desarrollo en las políticas...

\section{INTRODUCCIÓN}

Desde hace más de veinte años, se ha llegado a un cierto consenso de que la pobreza es uno de los principales elementos que define la disparidad entre países. Para medirla se tomó como punto de partida el número de personas que en 1990 vivían con menos de un dólar al día. Este indicador que mide la pobreza exclusivamente a partir de los ingresos económicos se discutió hace tiempo y aún aparece cuestionado en los debates de numerosos especialistas en desarrollo (Cornago, 1998; Barbé, 2007; Sanahuja, 2007; Unceta, 2009; Sotillo, 2011).

De hecho existen estimaciones de pobreza que se basan en otros indicadores tales como la desnutrición, la mortalidad infantil o el acceso a los servicios básicos, caso del Índice de Desarrollo Humano (IDH).

Dubois (2005) nos aclara que en aquellos primeros momentos la pobreza y la desigualdad no planteaban una corresponsabilidad internacional definida, no solo porque se les daba una explicación económica sino porque no se reconocía la necesidad de asumir colectivamente su superación.

Según las estadísticas del Banco Mundial relacionadas con la economía en el año 2008 vivían en el mundo alrededor de 6.700 millones de personas. En términos macroeconómicos una parte de la población, aproximadamente mil millones de personas, contaba con una Renta Per Cápita (RPC) de 67 euros al día. Sin embargo, la inmensa mayoría de la población mundial vivía, y sigue viviendo, en condiciones económicas precarias, que cruzan incluso el umbral de la pobreza extrema, ya que 4.600 millones de personas subsisten con una RPC diaria inferior a 7 euros y mil millones sobreviven con menos de 1,1 euros al día².

Este panorama de desigualdades ha interpelado a la sociedad internacional que ha llegado al consenso de que es urgente generar condiciones de desarrollo para todas las personas.

En este sentido, a finales de la década de los años ochenta la preocupación por los desequilibrios socioeconómicos llevó a que los Estados económicamente más desarrollados, a través de instituciones multilaterales tales como el BM y el Fondo Monetario Internacional (FMI), acordaran lo que se denominó el Consenso de Washington, iniciativa para el desarrollo global basada en políticas económicas neoliberales centradas en la liberalización de los mercados (Rist, 2002; Sanahuja, 2007; Sotillo, 2011). El pensamiento que subyacía a este planteamiento era que la no intervención de los gobiernos en las economías serviría como incentivo a la iniciativa privada, lo que promovería la creación de empleo y el consiguiente aumento del Producto Interior Bruto (PIB) dando como resultado la reducción de la pobreza. Es decir, partía de la premisa de que el mercado era la institución clave para conseguir el crecimiento y

2 Más información en http://datos. bancomundial.org/income-level/LIC [Actualizado en octubre de 2011]. 
la fuerza impulsora de este era el sector privado (Dubois, 2005).

En definitiva, el desarrollo consistía básicamente en conseguir que los países más pobres se acercaran a las pautas de los países más ricos; siendo la gran propuesta la identificación del desarrollo con el crecimiento económico.

Veinte años después la realidad mostró el fracaso de la iniciativa. Según el Informe de Desarrollo Humano del Programa de las Naciones Unidas para el Desarrollo (2009), las poblaciones de 54 países que se comprometieron con planes de desarrollo promovidos en el marco del Consenso de Washington, eran más pobres que antes de aplicarlos. Además, se percibía que los países pobres eran más dependientes de los industrializados y estaban obligados a aceptar sin discusión condiciones comerciales, tasas de interés y reglas de los préstamos, y criterios de uso de los fondos de ayuda internacional al desarrollo, entre otros (Sanahuja, 2007). Como menciona Unceta (2009: 27) «la tradicional expresión monetaria de los avances y retrocesos en términos de desarrollo, asociándolos únicamente con variables como el PIB, el consumo, el ahorro, o la inversión, resulta completamente insatisfactorias cuando se pretende adoptar un enfoque multidimensional», como corresponde con el siguiente compromiso que asumió la sociedad internacional.

En el 2000 esta se comprometió con una nueva iniciativa: los Objetivos de Desarrollo del Milenio (ODM) ${ }^{3}$.

3 En septiembre del 2000 se celebró en la sede de la Naciones Unidas en Nueva York
Fue promovida desde el ámbito de las Naciones Unidas, alentada con el ideal de un horizonte de futuro compartido por toda la humanidad y como intento coordinado de la sociedad internacional para promover el desarrollo en países con economías precarias. Este proceso

lo que se llamó la Cumbre del Milenio. Allí 189 jefes de Estado y de Gobiernos convinieron en establecer una serie de compromisos mensurables y con plazos definidos (Declaración del Milenio), para combatir la pobreza, el hambre, las enfermedades, el analfabetismo, la degradación del ambiente y la discriminación contra la mujer. En el 2001 se sistematizaron estos compromisos y se les dio el nombre de Objetivos de Desarrollo del Milenio. La fecha para alcanzar los ODM es 2015. Son ocho: 1. Erradicar la pobreza extrema y el hambre; 2. Lograr la enseñanza primaria universal; 3. Promover la igualdad de género y el empoderamiento de la mujer; 4. Reducir la mortalidad infantil; 5. Mejorar la salud maternal; 6. Combatir el SIDA, el paludismo y otras enfermedades; 7 . Garantizar la sostenibilidad del medio ambiente; 8. Fomentar una alianza mundial para el desarrollo. Ha habido algunos avances, pero los informes de seguimiento insisten en que queda mucha tarea por hacer. Los logros en algunos países demuestra una rápida consecución de los ODM cuando se combinan liderazgo de los gobiernos, políticas y estrategias de inversión pública en áreas vitales con ayuda adecuada, financiera y técnica de la Sociedad Internacional. Sin embargo, la carencia de un aumento significativo en la Ayuda Oficial al Desarrollo (AOD) desde el año 2004, hace imposible, incluso para los Países en Vías de Desarrollo (PVD) con gobiernos comprometidos, alcanzar los ODM. Más información en http:/www. un.org/milleniumgoals/pdf/mdg2007.pdf [Actualizado en octubre de 2011]. 
Nerea Azkona • La implementación del concepto de desarrollo en las políticas...

que se inició a comienzo del presente siglo es importante porque va a determinar la evolución de las políticas de desarrollo y, consecuentemente, las de cooperación internacional.

En la base de esta nueva iniciativa está la intención de que países con economías menos y más desarrolladas trabajen juntos con el horizonte de un futuro compartido 4 . Esta noción de desarrollo más integral que lo meramente económico, está en la línea de la propuesta del Premio Nobel de Economía de 1998, Amartya Sen, que considera que «el proceso de desarrollo de las libertades humanas incluye capacidades elementales como la capacidad de evitar hambrunas, desnutrición, mortalidad infantil, así como libertades que derivan de la alfabetización, de libertad de expresión, de la participación política» (Sen, 1999: 36).

En cuanto a la financiación de esta iniciativa en el año 2002, y también en el ámbito de Naciones Unidas, se convocó a una Conferencia Internacional en Monterrey (México) para confirmar los compromisos de financiación para el desarrollo que los Estados con economías más sólidas asumirían para la consecución de estos fines. Se aludió al objetivo de destinar a la Ayuda Oficial al Desarrollo el 0,7\% del PIB en el plazo

4 Cabe reseñar que los objetivos de la Cumbre del Milenio coincidieron con los que el CAD planteó en el año 1996 para los países donantes. Por lo cual, la fijación de los ODM no es el resultado de un diálogo entre países ricos y pobres, sino la aceptación por estos últimos de lo que ya habían acordado los primeros. de tiempo más breve posible. Seis años después (2008), se celebró en Doha (Qatar) una Conferencia de seguimiento de los compromisos del Consenso de Monterrey. Uno de los avances más significativos fue la decisión de los Estados miembros de la Unión Europea de aportar fondos para la AOD hasta alcanzar los ratios de $0,56 \%$ del PIB en 2010 y el $0,7 \%$ en $2015^{5}$.

\section{LA EVOLUCIÓN DEL}

\section{CONCEPTO DE DESARROLLO:}

\section{DEL CRECIMIENTO ECONÓMICO}

\section{AL DESARROLLO HUMANO}

El desarrollo es un término que no puede definirse de manera atemporal, ya que es la historia la que lo llena de contenido. Cada sociedad y cada época tienen su propia formulación de qué es el desarrollo, respondiendo a las convicciones, expectativas y posibilidades que predominan en ellas ${ }^{6}$.

A pesar de que la Unión Europea en 2010 fue el mayor donante mundial de AOD con 53.800 millones de euros $(0,43 \%$ del PIB), esta cifra no fue suficiente para alcanzar el objetivo de llegar al 0,56\% del PIB durante ese año. Para ver más en: http://europa.eu/rapid/press-release MEMO-11-221_en.htm?locale=en [Actualizado en abril de 2013].

6 Véase el vocablo «Desarrollo» elaborado por DUBOIS, A. en Pérez Alonso de Armiño, K., Diccionario de Acción Humanitaria y Cooperación al Desarrollo. 2000. Bilbao: Icaria y Hegoa. En Internet: http://www.dicc.hegoa.ehu.es/listar/ mostrar/67 [Actualizado en enero de 2013]. 
Al término de la II Guerra Mundial (1945) el mundo desarrollado se percató de la situación en la que se encontraban millones de seres humanos que vivían en zonas con bajos niveles técnicos, sanitarios, educacionales o económicos. Esta toma de conciencia fue acompañada por numerosas teorías que pretendían diagnosticar la situación para aplicar políticas de desarrollo adecuadas en las zonas afectadas.

El término subdesarrollo fue acuñado por el presidente estadounidense Harry Truman en 1949, que lo consideró un escalón hacia el desarrollo, de evolución lineal ascendente que, como han apuntado otros teóricos más adelante, nunca ha tenido lugar (Neef et alia., 1986; Sen, 1999; Rist, 2002).

Es decir, a partir de finales del decenio de 1940 más de 2 mil millones de habitantes del planeta llamados africanos, latinoamericanos y asiáticos cambiaron de nombre para pasar a denominarse simplemente "subdesarrollados» (Rist, 2002). Según palabras de Harry Truman el deber de los países desarrollados era ayudar económica y financieramente al resto de los pueblos para que consiguieran la estabilidad económica y el orden político. El subdesarrollo es, en este sentido, la «expresión de una escasa capacidad productiva y de un débil crecimiento económico» (Unceta, 2009: 9).

La citada perspectiva del presidente estadounidense era y es una visión realista de las relaciones internacionales basada en la modernización y en la revolución industrial, que considera al subdesarrollo como una etapa previa al desarrollo. Este paradigma enfatiza el poder militar y el político, la seguridad y la supervivencia del Estado.

A este respecto, la teoría de la modernización, que reza que una población debe pasar por una serie de etapas que le permitirán convertirse de una sociedad tradicional a una moderna, inauguró, para muchos teóricos y elites mundiales, un período de certeza bajo la premisa de los efectos benéficos del capital, la ciencia y la tecnología. La moderna economía del desarrollo partió de una visión limitada al problema de identificar y cuantificar el crecimiento económico.

La teoría clásica del desarrollo de Adam Smith confiaba en la posibilidad de una expansión casi ilimitada de la capacidad productiva del sistema y consideraba que el bienestar de las personas depende de manera directa de la riqueza global de los países donde viven. Según Unceta (2009: 8) «la atención quedaba centrada en el Estado-nación, no solo como ámbito principal en el que tomaban cuerpo los procesos económicos y sociales, sino también como sujeto mismo de derecho». Es decir, el desarrollo humano era considerado como un subproducto del desarrollo estatal (Sutcliffe, 1995).

Puede considerarse que estas son las propuestas ortodoxas que han respondido al pensamiento dominante y que han guiado las políticas de los organismos multilaterales y los gobiernos del mundo occidental.

Sin embargo, valorar el desarrollo sólo en función de factores económi- 
Nerea Azkona • La implementación del concepto de desarrollo en las políticas...

cos da una idea poco acertada de la realidad, puesto que el desarrollo económico de un país no implica siempre una correcta distribución de la renta ni la consecución de otros logros necesarios, como el acceso a la educación o a la sanidad. Estos y otros parámetros similares empezaron a tenerse en cuenta para medir la calidad de vida de la ciudadanía.

En la década de los años setenta se produjo una revisión crítica de los planteamientos ortodoxos y se abrió un espacio de acercamiento entre los diferentes enfoques, planteando alternativas críticas a la visión oficial ( $\mathrm{Du}-$ bois, 2005). Los resultados esperados de desarrollo que se anunciaban desde las propuestas oficiales no se producían, dándose por el contrario en muchos países un incremento de la pobreza que cuestionaba los planteamientos basados exclusivamente en el crecimiento económico. Ante tal situación surgieron las llamadas teorías de la dependencia que fueron la contrapartida de las teorías de la modernización, elaboradas principalmente en los propios países subdesarrollados.

Sin embargo, en la década de los años ochenta se retornó a las posiciones anteriores bajo el denominado Consenso de Washington, hasta que en los años noventa el debate sobre el desarrollo experimentó un punto de inflexión al cuestionar el propio concepto de desarrollo un creciente número de estudios críticos en muchas partes del mundo. Estos críticos analizaban el desarrollo como un discurso de origen occidental que operaba como un poderoso mecanismo para la producción cultural, social y económica del Tercer Mundo (Escobar, 2005).

Para autores como Rist (2002) el desarrollo sería a la vez un producto de la historia y también una idea capaz de producir historia, condicionando decisivamente la evolución de las sociedades en todas las partes del mundo ${ }^{7}$.

Hasta entonces la visión del desarrollo venía marcada por la idea de la modernización como escenario por perseguir. Sin embargo, estas propuestas que entienden el desarrollo como un proceso lineal comienzan a resquebrajarse con las formulaciones del desarrollo sostenible y del desarrollo bumano. Este último cuestiona el concepto mismo de desarrollo que la sociedad tiene considerado como deseable. Implica, de la misma manera, la concepción del desarrollo como un proceso de expansión de las capacida-

7 Como instrumento que fue creado al servicio de esa idea, la maquinaria de la cooperación para el desarrollo constituye igualmente un conjunto de realidades de orden material que no podemos ignorar, en forma de flujos económicos, entramados institucionales, movimientos de personas, infraestructuras y otros muchos aspectos que resultan fáciles de identificar. Sin embargo, la cooperación para el desarrollo se configura también a partir de un conjunto de representaciones que sobre las condiciones del mundo, y sobre sus expectativas de transformación, nos ofrecen constantemente los medios de comunicación, las instituciones internacionales, las agencias de desarrollo y los profesionales de la cooperación (Ávila Pacheco y Cornago, 2009). 
des de las personas, incluida la elección de su modo de vida.

En esta línea, Neef y otro. (1986) apostaron por un enfoque transdisciplinario que coloca a las ciencias económicas en diálogo con las ciencias sociales y humanas, en especial con la sociología, la antropología y la psicología. Trabaja un enfoque del desarrollo que privilegia una pedagogía de participación en la base y de autodependencia (independencia y autonomía) progresivas. Aún no se le ha puesto el adjetivo de "humano» al concepto de desarrollo.

No obstante, a partir de la crítica de Amartya Sen se abrió la puerta para una reformulación conceptual de la noción de bienestar que permitía otra caracterización del progreso. Es en este momento cuando la perspectiva del desarrollo bumano plantea según Unceta:

una importante ruptura conceptual con la manera convencional de entender el desarrollo, reivindicando la necesidad de un nuevo examen de medios y fines, la invalidez de los indicadores utilizados, el análisis en términos de procesos y no sólo de resultados, y la consideración de distintos ámbitos espaciales a la hora de estudiar los diversos aspectos que inciden en el bienestar humano (Unceta, 2009: 21).

Una característica común de estas revisiones críticas es el reconocimiento de que la lectura ha estado demasiado marcada por los intereses y percepciones de los países desarrollados. Se impone hacer una lectura histórica y concreta desde la realidad, que tenga más en cuenta los planteamientos de los países del Sur (Dubois, 2005).

Además, esta noción se relaciona con el rechazo de la modernidad como referencia y con el cuestionamiento del propio concepto (Cornago, 1998; Rist, 2002; Unceta, 2009).

En 1990 fueron las Naciones Unidas las que implementaron el concepto de desarrollo humano, definiéndolo como «el proceso por el cual las comunidades, las familias y las personas llegan a ser más fuertes y pueden disfrutar de vidas plenas y productivas, y ser menos vulnerables ante los desastres ${ }^{8}$. De este modo se construye el Indicador de Desarrollo Humano (IDH).

El IDH incluye nuevas perspectivas que no se habían tenido en cuenta en indicadores solamente relacionados con el progreso económico. En estos modelos ya obsoletos, pero muy presentes en la actual política neoliberal, no se tiene en cuenta el resultado de siglos de dominación y de explotación. Esto requeriría que los países ricos proporcionaran los recursos necesarios para introducir cambios estructurales en los países más pobres mediante la ejecución de acciones de cooperación internacional que, aunque por sí solas no sean la panacea, permitieran lograr la erradicación del subdesarrollo, contribuyendo a paliar algunas de sus causas y efectos, como por ejemplo

8 Más información en http://hdr.undp.org/ es/desarrollohumano/ [Actualizado en octubre de 2011]. 
Nerea Azkona • La implementación del concepto de desarrollo en las políticas...

podrían ser los migrantes económicos (Fanjul y Ladra, 2006) .

La novedad del IDH fue la combinación de indicadores de esperanza de vida, logros educacionales e ingresos, complementando la visión económica con un punto de vista social. Al contrario de la perspectiva evolucionista del desarrollo, este índice apuesta por los individuos, los que son considerados la verdadera riqueza de los Estados.

Cada dimensión se valora, a su vez, a partir de unos subíndices. Estos permiten medir el avance de los parámetros establecidos respecto de unos niveles mínimos que se consideran necesarios para que una sociedad sobreviva en el tiempo. Los cálculos tienen una periodicidad de cinco años para analizar las

$9 \quad$ En este sentido y como consecuencia del llamado subdesarrollo, a lo largo de la historia la humanidad ha resuelto gran parte de sus problemas económicos recurriendo a los movimientos migratorios. En la actualidad, vemos crecer el número de migrantes económicos que recalan en los países ricos, procedentes de los países más empobrecidos. En una situación de equilibrio a nivel global, las personas no tendrían que abandonar sus lugares de origen y podrían alcanzar en ellos los niveles de vida a los que justamente aspiran. Existen otras explicaciones además de la migración económica que analizan las migraciones internacionales como por ejemplo la Teoría de Redes o el Transnacionalismo. Para ver más: RUIZ BALZOLA, A., 2005. "Transnational migratory spaces: the emergent of a new context for public policies» en SETIÉN, M.L. y VICENTE, T. (ed.), Crossdisciplinary Views on migration Diversity. Bilbao: University of Deusto. tendencias a largo plazo en el desarrollo humano.

El IDH mide el avance promedio de un país a partir de tres factores: la esperanza de vida al nacer (salud); los años promedio de instrucción y los años de instrucción esperados (educación); y el ingreso nacional bruto per cápita (estándar de vida). Estas dimensiones se fijan, a su vez, en: la mortalidad infantil y materna; la tasa de alfabetización de los adultos y la tasa bruta combinada de matriculación en enseñanza primaria, secundaria y terciaria; y el PIB per cápita.

En 2010 se introdujeron tres nuevos parámetros relacionados con la distribución del bienestar en la desigualdad, la equidad de género y la pobreza ${ }^{10}$. Además, se corrigieron aspectos del IDH que se habían criticado, aunque se mantuvieron las tres dimensiones originales: una vida saludable; el acceso a la educación; y un nivel de vida digno.

10 Puesto que las diferencias de género apenas se evalúan con el IDH, un indicador específico se encarga de esta cuestión. El Índice de Desigualdad de Género (IDG) analiza la desventaja de la mujer en tres dimensiones: la salud reproductiva; el empoderamiento; y el mercado laboral. Las variables que se tienen en cuenta para este indicador son cinco: la mortalidad materna y la fecundidad adolescente relacionados con la salud reproductiva; los escaños en el Parlamento y el nivel de instrucción vinculados al empoderamiento; y la participación en la fuerza laboral relacionado con el mercado laboral. Sin embargo, ni la propiedad de bienes, ni la violencia de género, ni la participación en la toma de decisiones comunitarias quedan reflejados en este índice. 
3. LA COOPERACIÓN AL

DESARROLLO: CONCEPTO Y

EVOLUCIÓN

\subsection{Definición y paradigmas \\ a partir de las relaciones internacionales}

En sus inicios el sistema internacional de cooperación y ayuda al desarrollo tuvo en los Estados y en las organizaciones intergubernamentales sus principales y casi únicos protagonistas. Sin embargo, a lo largo de los decenios de 1960 y 1970 la sociedad civil se incorporó a dicho sistema a través de las organizaciones no gubernamentales de desarrollo. También lo hicieron los gobiernos no centrales (regiones, comunidades autónomas...) y los Gobiernos Locales (ciudades y municipios).

Nos encontramos frente a un sistema plural y descentralizado, ya que está formado por multitud de organizaciones de distinta naturaleza (públicas y privadas). Esto no significa que ni el sistema internacional ni la cooperación al desarrollo sean anárquicos, ya que ambas están institucionalizadas y reguladas (Fanjul y Ladra, 2006; Gómez Galán y Sanahuja, 1999).

La cooperación para el desarrollo puede definirse como «un conjunto de actuaciones, realizadas por actores públicos y privados, entre países de diferente nivel de renta con el propósito de promover el progreso económico y social de los países del Sur de modo que sea más equilibrado en relación con el Norte y resulte sostenible» (Gómez
Galán y Sanahuja, 1999: 17) ${ }^{11}$. Ese conjunto de acciones de carácter internacional está orientado al intercambio de experiencias y recursos entre países del Norte y del Sur para alcanzar metas comunes basadas en criterios de solidaridad, equidad, eficacia, interés mutuo, sostenibilidad, corresponsabilidad y coherencia (Sotillo, 2011) ${ }^{12}$. El fin primordial es erradicar la pobreza, el desempleo y la exclusión social; buscar la sostenibilidad y el aumento permanente de los niveles de desarrollo político, social, económico y cultural en los países del Sur ${ }^{13}$. A partir de estos objetivos hemos realizado el baremo de coherencia e incoherencia de otras políticas para con la cooperación.

Las actuaciones de la cooperación al desarrollo se sitúan en el marco de las relaciones internacionales, y están dirigidas a mejorar las condiciones de vida de la población de los países de menos renta relativa, impulsando el

11 No debemos olvidar el intercambio de recursos, tecnología y conocimiento entre Países en Vías de Desarrollo, lo que se denomina Cooperación Sur-Sur.

12 Dentro de este esquema, la AOD es sólo uno de los macro-instrumentos de las políticas públicas de cooperación. El sistema de cooperación va más allá de la ayuda y puede haber colaboración en distintos ámbitos cuyo fin no es el desarrollo.

13 Véase el vocablo «Cooperación para el desarrollo» elaborado por DUBOIS, A. en Pérez Alonso de Armiño, K., Diccionario de Acción Humanitaria y Cooperación al Desarrollo. 2000. Bilbao: Icaria y Hegoa. En Internet: http://www.dicc.hegoa.ehu. es/listar/mostrar/44 [Actualizado en enero de 2013]. 
Nerea Azkona • La implementación del concepto de desarrollo en las políticas...

crecimiento económico y el bienestar social, favoreciendo formas de gobierno democráticas y contribuyendo a la preservación del medio ambiente. Además, existe el criterio del interés mutuo como componente real de la cooperación, ya que también se derivan beneficios para el país que aporta los recursos (Gómez Galán y Sanahuja, 1999).

Como ya se ha mencionado, la cooperación al desarrollo nace después de la II Guerra Mundial como resultado de las necesidades de reconstrucción, organización política, económica y social de Europa y los nuevos Estados independientes. Comienza a desarrollarse en pleno debate entre la corriente realista de la disciplina de las relaciones internacionales, que va cogiendo más fuerza, y la idealista, que al volver a repetirse otra situación de conflicto extremo, como fue la II Guerra Mundial, perdió credibilidad (Sodupe, 2003).

Desde las perspectivas (neo)realistas y (neo)liberalistas las motivaciones de la cooperación al desarrollo responden a intereses estatales a corto y a largo plazos, en el primer caso; y resaltan las necesidades vinculadas a motivaciones más o menos altruistas relacionadas con la lucha contra la pobreza, la ayuda humanitaria y de emergencia y los intereses compartidos, en el segundo.

Por lo tanto, podríamos alinear las teorías realistas e institucionalistas, cuyos autores de referencia son Hans Morguentau, Kenneth Waltz, Joseph Nye y Robert Keohane, ya que ambos paradigmas están más vinculados a las grandes potencias, tendiendo a justificar y/o racionalizar las tradiciones políticas de intervención de las grandes potencias en los países de la periferia (Espinoza, 2003). Aunque existen diferencias significativas.

Para los realistas y los neorrealistas el campo de estudio se construye alrededor del poder político. Los Estados son los únicos actores significativos ya que son los que lo poseen y los que actúan racionalmente calculando los costos y beneficios de sus actuaciones en la búsqueda de aumentar su poder o de evitar que disminuya, apoyándose en conceptos clave como la balanza de poder y el interés nacional. En este caso, la cooperación queda en función de la maximización del poder de los Estados.

En cambio, para los institucionalistas lo importante en las relaciones internacionales es la interacción entre los diversos actores que actúan en el plano mundial, lo que ayuda a desarrollar la idea de un sistema internacional de relaciones y de un régimen internacional, donde la cooperación, como relación, es vista de otra manera.

Como apunta Baldwin (1993) los neorrealistas ven la cooperación internacional difícil de alcanzar, de mantener y más dependiente del poder del Estado que los neoliberales institucionalistas. Esto se debe a que para los primeros la anarquía que caracteriza las relaciones internacionales, dada la ausencia de un Estado mundial, es lo que inhibe la cooperación, a partir del temor de los Estados a ser dominados, destruidos o engañados por otros más fuertes (Espinosa, 2003). Según Baldwin (1993), la 
posibilidad de que en la cooperación un Estado pueda tener ganancias desproporcionadas y que pueda convertirse en un amigo dominante o en un potencial adversario poderoso es lo que inhibe la cooperación.

Para los institucionalistas, en contra, lo fundamental son las ganancias absolutas que cada Estado obtiene (Keohane, 1984). Argumento que no resultaría satisfactorio para los Estados del Sur ya que las ganancias relativas lo son aún más dadas las desigualdades internacionales existentes.

\subsection{La evolución de las perspectivas sobre la cooperación al desarrollo}

Este tipo de colaboración ha atravesado periodos de transformación a causa de las modificaciones que ha experimentado la sociedad internacional a lo largo de los años y como resultado de las perspectivas diferentes de desarrollo que manejan las diferentes teorías académicas.

La cooperación al desarrollo es un concepto dinámico, resultado de una construcción histórica directamente relacionada con el concepto y las teorías sobre la noción y la práctica del desarrollo que se han ido elaborando paralelamente, sufriendo cambios en función del pensamiento, políticas y valores presentes en las relaciones Norte-Sur y Sur-Sur.

En los años sesenta y setenta el debate osciló entre las teorías de la modernización y el llamado «enfoque de la dependencia». En los años ochenta retornó el neoliberalismo y emergieron nuevas perspectivas, como son el desarrollo humano, el desarrollo sostenible o el enfoque de género, que han dado origen a una vasta producción bibliográfica. En la década de los noventa se puso en cuestión el propio concepto de «desarrollo» por nuevas teorías críticas de matriz deconstructivista, como el postdesarrollo. El fenómeno de la globalización y la crisis del neoliberalismo han dado paso a nuevos enfoques críticos.

Las ideas desarrollistas de las décadas de los años cincuenta y sesenta, basadas en el crecimiento económico como objeto central y en la confianza de un crecimiento ilimitado, hicieron de la cooperación al desarrollo un instrumento dependiente de las estrategias económicas.

Además, fueron clave la Guerra Fría y las relaciones bilaterales que a partir de esta asume Estados Unidos con terceros países para atraerlos a su esfera de influencia política y económica, así como el impulso de las políticas oficiales de cooperación bilateral que algunos países europeos orientaron principalmente hacia sus excolonias fueron claves (Sotillo, 2011).

Esta concepción de la cooperación, dominada por los países donantes, no se caracterizó por la igualdad y la colaboración mutua, sino que fue entendida más como una iniciativa voluntaria y generosa de éstos que como una obligación hacia los receptores. La idea de donación implicaba la no obligatoriedad y establecía una posición de inferioridad 
Nerea Azkona • La implementación del concepto de desarrollo en las políticas...

por parte de quien recibía la ayuda. De hecho, la carencia de una colaboración real entre los países donantes y receptores adquiere todo su significado en la existencia y funcionamiento de diversas formas de condicionalidad de la ayuda, que forman parte sustancial de la cooperación (Fanjul y Ladra, 2006).

El decenio de 1970 estuvo marcado por cambios económicos muy importantes que afectaron a las relaciones Norte-Sur y a la cooperación al desarrollo, que vivió en esta década un tiempo de balances, crisis y replanteamientos. Ya desde el final de la década anterior comenzó a debatirse con relativa intensidad sobre los efectos producidos por largos años de crecimiento económico (Unceta y Yoldi, 2000).

De este modo, nos encontramos con las teorías de la dependencia y del desarrollismo, que forman una aproximación más crítica hacia el papel de las instituciones internacionales como factores de dominación y generadores de dependencia. Para estos teóricos, lo que caracteriza a las relaciones internacionales entre los países del Norte y del Sur es la explotación y la dominación (Sotillo, 2011). Conciben la cooperación internacional como posible entre los países del Sur con estrategias de desarrollo orientadas hacia el cambio de las relaciones internacionales estableciendo nuevas reglas. La mayor crítica a las teorías del realismo y del idealismo es que las diferencias no son sólo cuantitativas sino que también son cualitativas. Estas últimas son de carácter estructural, "diferencias generadoras de relaciones de dependencia, capaces de dificultar, impedir o estrangular el crecimiento económico, pudiendo llegar a bloquear el proceso de desarrollo» (Unceta, 2009: 10).

Los estudios realizados por los teóricos de la dependencia sirvieron de fundamento para las propuestas de un nuevo orden internacional y la reformulación de estrategias de desarrollo que rompieran la dependencia existente entre países. Según Espinosa (2003) esta teoría ha sentado las bases de las vías de cooperación internacional para reducir o evitar la dependencia mediante cambios estructurales. Esto se debe a que conciben las relaciones internacionales como un sistema en el que las potencias centrales conforman un orden mundial adecuado a sus intereses con instituciones internacionales controladas por dichos países dirigidas a conservar las formas de dominación internacional.

Por su parte, el desarrollismo, planteado sobre todo por la Comisión Económica para América Latina y el Caribe (CEPAL), se ha caracterizado por el diagnóstico sobre la dinámica centroperiferia y las propuestas de políticas de desarrollo ${ }^{14}$. La cooperación internacional constituye un instrumento para lograr el desarrollo, concibiendo como posibles y necesarias la cooperación Norte-Sur, la Norte-Norte y la Sur-Sur, algo sugerente y nuevo.

Según Hirschman (1980) la principal aportación de estas corrientes

14 Más información en http://www.eclac.cl/ cooperacion/ [Actualizado en octubre de 2011]. 
críticas fue la negación del beneficio mutuo; sin embargo, no cuestionaron que el crecimiento económico fuese la principal y casi única herramienta para salir del subdesarrollo. Es decir, «la impugnación de la ortodoxia no vino a cuestionar la cada vez mayor identificación del desarrollo con el crecimiento económico» (Unceta, 2009: 10).

La corriente postmoderna se acerca a la concepción del desarrollo desde la sostenibilidad y el desarrollo humano y se caracteriza por el activismo de principios (Sotillo, 2011). Sus teóricos se dieron cuenta del error de haber asumido una relación automática entre crecimiento y bienestar, hasta el punto de hacer del primero la referencia central de la estrategia, dejando en un segundo plano las necesidades e intereses vitales de la gente, así como las exigencias derivadas de la base de recursos existente (Unceta, 2009). Esta idea que nos invita a superar la dicotomía conceptual desarrollo-subdesarrollo hace que nos cuestionemos las políticas de desarrollo que deben ser acompañadas de cambios profundos en la distribución mundial del poder y de los recursos.

De este modo, al terminar la década de los años noventa existía un consenso sobre la necesidad de revisar los esquemas de la colaboración. Por una parte, los factores de la Guerra Fría y de la descolonización han dejado de existir; por la otra, se dan otros dos procesos diferentes: la quiebra del pensamiento del desarrollo; y el fenómeno de la globalización.

En cuanto al primero, la hegemonía del neoliberalismo tiene consecuencias directas sobre las estrategias encaminadas a alcanzar el desarrollo. Dado que su pretensión es liberar al máximo las fuerzas del mercado, considera que la cooperación al desarrollo no sirve para promover los factores dinámicos del desarrollo y que, por el contrario, puede ser un elemento perjudicial por suponer una interferencia en el buen funcionamiento de los mecanismos del mercado. Es decir, la cooperación debería reducirse al mínimo o limitar su campo de actuación actual ${ }^{15}$.

Sin embargo, el modelo de cooperación al desarrollo imperante en la actualidad se basa en el desarrollo humano, que entiende que es un proceso de expansión de las capacidades de las personas, de manera que puedan elegir su modo de vida (Sotillo, 2011). Se sustituye la visión del desarrollo centrado en la producción de bienes por otra relacionada con la ampliación de las capacidades de las personas.

Por su parte, la globalización ha generado cambios orgánicos en las dinámicas de poder que estructuran las relaciones entre los diferentes Estados, actores, instituciones y personas. Este proceso se aceleró con la caída del Muro de Berlín «porque la desaparición de la barrera ideológica bipolar permitió la entrada de más jugadores que intentaron adaptarse a las reglas existentes para evitar la exclusión» (García Segura, 1999: 349).

15 Vocablo "Cooperación para el desarrollo", DUBOIS, A. en Pérez Alonso de Armiño, K., Diccionario de Acción Humanitaria y Cooperación al Desarrollo, op. cit. 
Nerea Azkona • La implementación del concepto de desarrollo en las políticas...

Si bien históricamente los fenómenos de interdependencia han sido elementos relevantes en las dinámicas de la sociedad internacional es a partir de la segunda mitad del siglo XX cuando estas adquieren un carácter transformador y a la vez constituyente del proceso de globalización (Arenal, 2009). Estos han reconfigurado la naturaleza de las relaciones de poder entre los Estados (Arenal, 2009); y dichos fenómenos asimétricos se constituyen en una importante fuente de poder (Arenal, 2002).

A medida que el mundo se vuelve progresivamente interdependiente, los problemas del desarrollo adquieren una dimensión global que afecta, inevitablemente, a todas las economías del planeta (inclusive las más ricas y desarrolladas). Junto con los procesos de progresiva interdependencia convergen altos niveles de desigualdad, lo que supone un importante riesgo para la sostenibilidad del sistema global (Millán, 2012: 103).

La globalización no es un fenómeno mundial a corto plazo, puesto que es un proceso todavía desigual y parcial y no existen signos de que la homogeneización económica, social y cultural vaya a alcanzarse (García Segura, 1999).

Además, este fenómeno se ha producido bajo la hegemonía del neoliberalismo, que según los datos insiste en la agudización de las desigualdades, la aparición de nuevos procesos de empobrecimiento y la resistencia de la pobreza a disminuir, aun cuando haya aumentado el producto bruto mundial.

\section{LA COOPERACIÓN EN} MATERIA MIGRATORIA Y SU DIMENSIÓN EXTERIOR

La migración es un tema complejo y multifacético que afecta a todos los países del mundo. Casi todos son lugares de origen, tránsito o destino de personas migrantes, cuando no son las tres cosas a la vez. Un enfoque conjunto y proactivo entre los distintos países es muy importante para la gestión de la migración internacional.

En el contexto actual las migraciones internacionales son en gran parte resultado de las fuertes desigualdades en términos de desarrollo y poder entre unos países y otros, enmarcándose de este modo en las relaciones Norte-Sur.

Si concebimos las migraciones como el fenómeno integral que son, debemos asumir la responsabilidad que tienen a este efecto las políticas de cooperación internacional.

\subsection{La dimensión exterior de la política migratoria europea}

El binomio migraciones y desarrollo ha entrado de lleno en la agenda política de los países más ricos, que se enfrentan a la necesidad de adoptar un discurso que legitime su decisión de controlar las fronteras del Norte (Carballo de la Riva y Echart, 2007). Es por ello que las migraciones han de entenderse dentro de un contexto mundial de creciente interdependiencia, lo que requiere de un enfoque global y 
coherente que incluya todas las dimensiones de éste fenómeno.

Debemos abordar el tema de las migraciones y el desarrollo desde una perspectiva política ya que la incidencia de estas se ha introducido en el debate internacional y europeo como eje central de la actual agenda de política exterior. De hecho, según Malgesini:

El discurso predominante [de las NN.UU., la OIM y la UE] consiste en afirmar que el desarrollo de los países de origen contribuirá a frenar la emigración al atacar sus causas profundas: crisis e inestabilidad económica, desempleo, bajo nivel de crecimiento, bajo nivel de democratización política, conflictos sociales, elevado crecimiento vegetativo, etc. La cooperación internacional cumpliría la función de potenciar un mayor desarrollo y, nsecuentemente, actuaría como un mecanismo inhibidor de las emigraciones hacia los países de recepción, que son los mismos que los donantes de la ayuda. En suma, el lema sería 'dar más cooperación para tener menos inmigración’́ (Malgesini, 2005: 76).

Efectivamente, a partir del año 2005 en el ámbito europeo hubo una modificación del enfoque de las migraciones que consiguió ampliar la cooperación al desarrollo a una parte de la política migratoria, la llamada dimensión exterior.

Este enfoque global es una innovadora forma de tratar la gestión de la migración, cuyo objetivo es formular un conjunto amplio y coherente de políticas que no solo aborde una serie de asuntos relacionados con la migración sino también aúne asuntos de justicia y política interior, desarrollo y relaciones exteriores en un esfuerzo por mejorar el diálogo y la cooperación sobre la migración en asociación estrecha con terceros países.

En esta línea están redactadas las Conclusiones de la Presidencia del Consejo Europeo de Bruselas de los días 15 y 16 de diciembre de 2005, en el que se dejó constancia de «la necesidad de contar con un enfoque equilibrado y coherente, que abarque medidas orientadas a luchar contra la inmigración ilegal y aproveche, en cooperación con terceros países, los beneficios de la migración legal» (Unión Europea, 2005: 2).

La idea del enfoque en palabras de la Comunicación Reforzar el Planteamiento Global de la Migración: aumentar la coordinación, la coherencia $y$ las sinergias (COM(2008) 611) de la Comisión Europea:

Se basa en una auténtica asociación con los terceros países, está plenamente integrada en otras políticas externas de la UE, y trata todas las cuestiones de migración y asilo de manera global y equilibrada. Adoptado en 2005, el planteamiento ilustra la ambición de la UE de establecer un marco intersectorial para gestionar la migración de manera coherente a través del diálogo político y la estrecha cooperación práctica con los terceros países (Comisión Europea, 2008: 2).

Sin embargo, como señala Carballo de la Riva:

La aplicación del enfoque global de manera desigual en función de áreas 
Nerea AzKona • La implementación del concepto de desarrollo en las políticas...

geográficas e intereses divergentes, remarca las tensiones constantes entre los distintos pilares del enfoque. Dichas tensiones serán uno de los grandes retos que existen en la actualidad en la agenda del desarrollo en un ámbito como es el de la coherencia de políticas (Carballo de la Riva, 2009: 22).

En los documentos elaborados a partir del enfoque global de las migraciones se percibe la posibilidad de abordar migraciones y cooperación al desarrollo de manera conjunta, para cumplir con la exigencia de una mayor coherencia de políticas. Sin embargo, por otra parte, se ve la cooperación, en la medida que fomenta el desarrollo de la zona de origen de la emigración, como una herramienta que puede contribuir a frenar los flujos migratorios. Ambas perspectivas han promovido medidas que suscitan el control de fronteras en origen y la inclusión de la cooperación para el desarrollo en las estrategias migratorias. Los acuerdos de nueva generación firmados al amparo de los Planes África son ejemplos concretos de estas prácticas.

Por lo tanto, nos encontramos ante el riesgo de un planteamiento que avanza en la ejecución de una política migratoria que se sirve de las políticas de cooperación para frenar las migraciones, a través de la Ayuda Oficial al Desarrollo. Por ello, hay que analizar los planes de cooperación y el tratamiento que se está dando a ciertos países de África Subsahariana como los países de África Occidental, considerados frontera sur de Europa, y a los que desde la Unión Europea y desde los Estados miembros se está responsabilizando del control de los flujos migratorios (Carballo de la Riva, 2009) ${ }^{16}$.

De este modo la gestión de las migraciones entra de lleno en las agendas de las relaciones exteriores de los Estados y en las negociaciones internacionales. Entre los elementos e instrumentos que vinculan las políticas exteriores con las migratorias encontramos las relaciones con terceros países, los acuerdos de readmisión, el control de flujos y el vínculo de las migraciones con las políticas de desarrollo.

\subsection{La externalización de \\ la frontera exterior y la condicionalidad migratoria}

El concepto de externalización de la frontera se relaciona con el reforzamiento del control de los flujos, desplazando la frontera hacia los países del Sur con el objetivo de reducir las causas por las cuales los migrantes deciden abandonar su país. Esta categoría analítica está vinculada muy íntimamente con la dimensión exterior de las políticas migratorias. Se diferencian en que esta última se refiere a todos los aspectos de compromiso y política dirigidos al lado externo de sus fronteras. En realidad

16 El foco inicial del enfoque global de las migraciones fue África, afectada por alguno de los aspectos más negativos de la migración, como es: la fuga de cerebros, la inmigración ilegal o el tráfico de seres humanos. 
la noción de externalización se refiere a una variedad de prácticas políticas, y todas ellas están relacionadas con la extensión de la gobernación y de las políticas más allá de las fronteras.

De hecho, la externalización se ve acompañada por la securitización y la internacionalización de las políticas migratorias. Según Zapata-Barrero y Zaragoza Cristiani:

los distintos conceptos relacionados con la externalización contemplan, al menos, dos países diferentes que tienen una relación específica asimétrica, no sólo en términos de poder y de diferencias socio-económicas, sino que también en términos de capacidad de respuesta política para un mismo hecho: el movimiento de personas de un país a otro (Zapata-Barrero y Zaragoza Cristiani, 2008: 187).

A fin de cuentas, la externalización se define como un nuevo modo de gobernabilidad, ya que implica que la inmigración se está integrando como tema clave en las negociaciones internacionales y expresa, directa o indirectamente, la corresponsabilidad en un interés común: evitar el movimiento de personas. Es decir, la política de externalización lo que pretende es reducir los flujos sin tener que controlar las fronteras territoriales. Es una "política a distancia» y de conversión de la política de inmigración de un Estado en un instrumento de acción exterior.

En este marco, nos parece oportuno hablar de una "condicionalidad migratoria» que emerge en la política exterior y se constituye como pilar central de la delegación del control. Este proceso construye una relación cada vez más estrecha y ambigua entre la dimensión exterior de la política de inmigración y la Ayuda Oficial al Desarrollo. La emergente "condicionalidad migratoria", que instrumentaliza la ayuda, corre el riesgo de desvirtuar los objetivos básicos de la AOD (Gabrielli, 2012). Ésta es la renovada condicionalidad en el nuevo marco de la cooperación al desarrollo vinculada al fenómeno migratorio.

En este contexto se utilizan varios instrumentos de política exterior para superar los escollos en la negociación de esta externalización y para obtener una colaboración en materia de control de flujos migratorios. En particular, los acuerdos comerciales, las promesas de inversión, las cuotas migratorias y, sobre todo, las concesiones de AOD que son utilizadas como instrumentos de presión frente a terceros países (Gabrielli, 2012) $)^{17}$.

En concreto, los acuerdos de nueva generación (ANG) firmados con arreglo a los Planes África entre el Estado español y los países de África Occidental, son una amalgama de todos estos instrumentos, puesto que su objetivo último es el control de los flujos migratorios.

17 Estas prácticas vienen determinadas por una serie de actuaciones: el rechazo en la entrada del país de personas, el refuerzo del control en los países de tránsito, la intercepción de las embarcaciones antes de la llegada a tierra y los acuerdos de readmisión (Carballo de la Riva, 2009). 
Nerea Azkona • La implementación del concepto de desarrollo en las políticas...

Los ANG surgieron con la idea de abordar la cooperación en materia migratoria de un modo integral y coherente. De este modo, partían de la idea de que la inmigración se concibe como un fenómeno complejo y mediante estos instrumentos el Estado español pretendía ordenar este fenómeno en su globalidad, sustituyendo las herramientas que venían abordando parcialmente la gestión de este fenómeno. Así, los acuerdos de nueva generación no sólo hacen referencia a la regulación de los flujos migratorios laborales. Incorporan disposiciones en materia de lucha contra la inmigración ilegal, en general, y de readmisión de inmigrantes en situación administrativa irregular en uno de los Estados parte, en particular. También tienen cláusulas sobre la integración de los inmigrantes en la sociedad de acogida y sobre la potenciación de los vínculos entre la migración y el desarrollo. Esta era la teoría. En la práctica funcionan como instrumentos de repatriación dirigidos y negociados por el Ministerio de Asuntos Exteriores y Cooperación (MAEC) con condicionantes de la AOD.

\section{Conclusiones}

Tanto el término desarrollo como el de cooperación son dinámicos y resultado de una construcción histórica. Esta se vincula con los distintos períodos de transformación que ha experimentado la Sociedad Internacional y las diferentes perspectivas de desarrollo que se han ido manejando a lo largo del tiempo.

Según las propuestas, llamémoslas ortodoxas, sobre las teorías del desarrollo los problemas de la desigualdad y la pobreza estarían resueltos si las búsquedas de soluciones se limitaran a identificar y cuantificar el crecimiento económico. Sin embargo, valorar el desarrollo sólo en función de factores económicos da una idea poco acertada de la realidad, puesto que dicho desarrollo en un país no implica siempre una correcta distribución de la renta ni la consecución de otros logros necesarios, como el acceso a la educación o a la sanidad.

Por ello, este enfoque teórico está en consonancia con las formulaciones académicas y planteamientos políticos críticos de la visión meramente económica del desarrollo, abogando por incorporar otros factores para una adecuada consideración del desarrollo humano.

En 1990 fueron las Naciones Unidas las que elaboraron y propusieron el concepto de desarrollo bumano, apostando por los individuos, los cuales son considerados la verdadera riqueza de los Estados. Así, el modelo de cooperación al desarrollo predominante, en general, en la actualidad se basa en el desarrollo humano, que entiende que es un proceso de expansión de las capacidades de las personas, de manera que puedan elegir su modo de vida.

Por otra parte, el binomio migraciones y desarrollo ha entrado de lleno en la agenda política de los países más 
ricos, que se enfrentan a la necesidad de adoptar un discurso que legitime su decisión de controlar las fronteras del Norte. Es por ello que las migraciones han de entenderse dentro de un contexto mundial de creciente interdependencia, lo que requiere de un enfoque global y coherente que incluya todas las dimensiones de éste fenómeno.

En el momento en que los Estados consideraron las migraciones como un fenómeno global, asumieron la responsabilidad que tienen a este efecto las políticas de cooperación internacional. Así, han elaborado políticas de cooperación en las que subyacen dos ideas muy concretas:

- A más desarrollo en el país de origen de los inmigrantes y receptores de ayuda; menos inmigración en el país receptor de personas y donante de ayuda. El discurso predominante de las administraciones del Norte consiste en afirmar que el desarrollo de los países de origen contribuirá a frenar la migración. La cooperación internacional, en este caso, cumpliría la función de potenciar un mayor desarrollo y, consecuentemente, actuaría como un mecanismo inhibidor de las migraciones hacia los países de destino.

- Ayudar a los que colaboran mediante un sistema de "condicionalidad migratoria». Es decir, subyugar la cooperación a la corresponsabilidad en temas de repatriación y control de fronteras.

Estas dos ideas se materializaron por parte de la Unión Europea y del
Estado español a partir del enfoque global de las politicas migratorias y de los acuerdos de nueva generación, respectivamente.

En 2005 en el ámbito comunitario europeo se decidió una modificación del enfoque de las migraciones que consiguió introducir la cooperación al desarrollo en una parte de la política migratoria, la llamada dimensión exterior. Este planteamiento fue una innovadora forma de tratar la gestión de la migración, cuyo objetivo es formular un conjunto amplio y coherente de políticas que no sólo abordara aspectos relacionados con la migración, sino también asuntos de justicia y política interior, desarrollo y relaciones exteriores. Se entendía desde la UE como un esfuerzo para mejorar el diálogo y la cooperación sobre la migración en asociación estrecha con terceros países. La aplicación de esta nueva orientación de manera desigual en función de áreas geográficas e intereses divergentes, remarca las tensiones constantes entre los distintos prismas del enfoque: la lucha contra la inmigración ilegal; el fomento de la migración legal; y el desarrollo de las sinergias entre migración y desarrollo. Dichas tensiones son uno de los grandes retos que existen en la actualidad en la agenda del desarrollo en un ámbito como es el de la coherencia de políticas.

Por su parte, los acuerdos de nueva generación suscritos por España surgieron con la consideración de abordar la cooperación en materia migratoria de un modo integral y coherente. De este 
Nerea Azkona • La implementación del concepto de desarrollo en las políticas...

modo, partían de la idea de que la inmigración se concibe como un fenómeno complejo y mediante estos instrumentos se pretendía ordenar este fenómeno en su globalidad, sustituyendo las herramientas que venían abordando parcialmente su gestión. Así, estos convenios con terceros no solo hacen referencia a la regulación de los flujos migratorios laborales. Incorporan disposiciones en materia de lucha contra la inmigración ilegal, en general, y de readmisión de inmigrantes en situación administrativa irregular en uno de los Estados involucrados. Esta era la teoría. En la práctica funcionan como instrumentos de repatriación dirigidos y negociados por el MAEC, el cual subordina la AOD a su firma y ejecución.

Consideramos, en este sentido, que al tratarse de políticas de cooperación al desarrollo, lo coherente sería que las ideas que estuvieran detrás de las políticas de cooperación fueran las siguientes:

- A más cooperación en el país de origen más desarrollo para que la opción de migrar, o no, sea una alternativa real y positiva a tener en cuenta.

- Colaborar con los países para que se ayuden a sí mismos, a fin de luchar de manera eficaz y como plan a largo plazo contra la pobreza.

De este modo, la incoherencia de políticas de desarrollo está relacionada con el asociacionismo interesado, esto es y refiriéndonos a las políticas de cooperación en materia migratoria, con la formulación de políticas con objetivos prioritarios de seguridad y de control de flujos migratorios ilegales, dejando de lado, o en el peor de los casos en el olvido, el objetivo solidario de la cooperación al desarrollo.

Por lo tanto, nos encontramos ante el riesgo de un planteamiento que avanza en la ejecución de una política migratoria que se sirve de las políticas de cooperación para frenar las migraciones a través de la Ayuda Oficial al Desarrollo. De este modo la gestión de las migraciones entra de lleno en las agendas de las relaciones exteriores de los Estados y en las negociaciones internacionales.

\section{BIBLIOGRAFÍA}

ARENAL, C. del, (2002), «La nueva sociedad mundial y las nuevas realidades internacionales: un reto para la teoría y para la política», en Cursos de Derecho Internacional y Relaciones Internacionales de Vitoria-Gasteiz 2001, Bilbao, Universidad del País Vasco, pp. 17-86. En Internet: http://www.ehu.es/rsosderechointernacionalvitoria/ponencias/pdf/2001/2001_1. pdf

ARENAL, C. del, (2009), «Mundialización, creciente interdependencia y globalización en las relaciones internacionales», en Cursos de Derecho Internacional y Relaciones Internacionales de Vitoria-Gasteiz 2008, Bilbao, Universidad del País Vasco, pp. 181-268. En Internet: http://www.ehu.es/ rsosderechointernacionalvitoria/ponencias/pdf/2008/2008_4.pdf

ÁVILA PACHECO, V. y CORNAGO, N., (2009), «Pensar y hacer el (post)desarrollo más allá de un mundo lineal», en Revista Española de Desarrollo y Cooperación, $n^{\circ} 24$, pp. 5-13. 
BALDWIN, D., (1993), «Neoliberalism, neorealism and World Politics», en BALDWIN, D. (ed.), Neorealism and Neoliberalism. The Contemporary Debate, Nueva York, Columbia University Press, pp. 3-25. BARBÉ, E., (2007), Relaciones Internacionales, Madrid, Tecnos.

CALDUCH, R., (1991), Relaciones Internacionales, Madrid, Ciencias Sociales Ed.

CARBALLO DE LA RIVA, M. y ECHART MUÑOZ, E., (2007), «Migración y desarrollo: hacia un marco teórico común y coherente», en Revista Española Desarrollo y Cooperación, $n^{\circ} 19$, pp. 53-68. En Internet: http://pendientedemigracion. ucm.es/info/IUDC/img/archivos/documentos/Carballo_y_Echart.pdf

CARBALLO DE LA RIVA, M., (2009), Migraciones y desarrollo: la incidencia de África subsahariana en la agenda política. Documento de Trabajo $\mathrm{n}^{\circ} 13$, Madrid, Instituto Universitario de Desarrollo y Cooperación (IUDC) y Universidad Complutense de Madrid (UCM). En Internet: http://pendientedemigracion. ucm.es/info/IUDC/img/articulos/publicaciones/MIGRACIONES_Y_DESARROLLO_DT13.pdf

COMISIÓN EUROPEA, (2008), Comunicación de la Comisión al Parlamento Europeo, al Consejo, al Comité Económico y Social Europeo y al Comité de las Regiones Reforzar el Planteamiento Global de la Migración: aumentar la coordinación, la coherencia y las sinergias, Bruselas, 8-10-2008 (COM(2008) 611 final). En Internet: http://ec.europa.eu/ development/icenter/repository/COMM_ PDF_COM_2008_0611_F_ES_COMMUNICATION.pdf

CORNAGO, N., (1998), «Desarrollo, subdesarrollo y postdesarrollo: un análisis crítico del debate contemporáneo", en Cursos de Derecho Internacional de Vitoria-Gasteiz 1997, Madrid y Bilbao, Tecnos y Universidad de País Vasco, pp. 39-88. En Internet: http://www.ehu.es/ rsosderechointernacionalvitoria/ponencias/pdf/1997/1997_2.pdf
DUBOIS, A., (2005), «La revisión del desarrollo a principios de siglo. Entre el nuevo consenso y la propuesta alternativa», en DUBOIS, A. et. alia, Tendencias de la cooperación para el desarrollo y futuro de las ONGD, Donostia/San Sebastián, Diputación Foral de Gipuzkoa y Gobierno Vasco, pp. 9-32.

ESCOBAR, A., (2005), «El 'postdesarrollo' como concepto y práctica social», en MATO, D. (coord.), Políticas de economía, ambiente y sociedad en tiempos de globalización, Caracas, Universidad Central de Venezuela, pp. 17-31.

ESPINOZA, E., (2003), La cooperación internacional en las relaciones internacionales de Cuba, Brasilia, Universidad de Brasilia.

FANJUL G. y LADRA, S., (2006), 50 años de pobreza, 50 años de desarrollo. Madrid: Intermón Oxfam. En internet: http://www. intermonoxfam.org/sites/default/files/ documentos/files/061009_50pobreza.pdf

GABRIELLI, L., (2012), «El co-desarrollo y la externalización europea del control migratorio hacia África: los riesgos de una relación ambigua», en ABOUSSI, $\mathrm{M}$. (ed.), El codesarrollo a debate, Granada, Comares Editorial, pp. 47-58.

GARCÍA SEGURA, C., (1999), «La globalización en la sociedad internacional contemporánea: Dimensiones y problemas desde la perspectiva de las Relaciones Internacionales», en Cursos de Derecho de Vitoria-Gasteiz 1998, Madrid y Bilbao, Tecnos y Universidad del País Vasco, pp. 319-350. En Internet: http:/www.ehu.es/ rsosderechointernacionalvitoria/ponencias/pdf/1998/1998_7.pdf

GÓMEZ GALÁN, M. y SANAHUJA, J.A., (1999), El sistema internacional de cooperación al desarrollo. Una aproximación a sus actores e instituciones, Madrid, CIDEAL.

HIRSCHMAN, A.O., (1980), "Auge y ocaso de la teoría económica del desarrollo", en El trimestre económico, $\mathrm{n}^{\circ} 188$, pp. 1055-1077.

KEOHANE, R.O., (1984), After Hegemony. Cooperation and Discord in the World 
Nerea Azkona • La implementación del concepto de desarrollo en las políticas...

Political Economy, Princeton, Princeton University Press (versión en castellano de 1988. Después de la Hegemonía: cooperación y discordia en la política económica mundial, Buenos Aires, GEL).

MALGESINI, G., (2005), «Entre la inmigración y la cooperación en España: ¿existe espacio para el codesarrollo?», en ZÚÑIGA GARCÍA-FALCES, N. (coord.), La migración. Un camino entre el desarrollo y la cooperación, Madrid, Consejería de Servicios Sociales de la Comunidad de Madrid y Centro de Investigación para la Paz, pp. 75-104.

MILLÁN, N., (2012), Trasnacionalización del desarrollo y coherencia de políticas. Un análisis de los casos de España y Suecia, Tesis Doctoral, Facultad de Ciencias Políticas y Sociología de la Universidad Complutense de Madrid. En Internet: http://eprints.ucm.es/20057/1/T34324.pdf

NEEF, M., ELIZALDE, A. y HOPENHAYN, M., (1986), Desarrollo a Escala Humana. Una opción para el futuro, Santiago de Chile, El Centro de Alternativas de Desarrollo- CEPAUR y Fundación Dag Hammarskjold.

PÉREZ ALONSO DE ARMIÑO, K. (dir.), (2000), Diccionario de Acción Humanitaria y Cooperación al Desarrollo, Bilbao, Icaria y Hegoa. En Internet: http://www. dicc.hegoa.ehu.es/

PNUD, (2009), Informe sobre desarrollo humano 2009. Superando barreras: Movilidad y desarrollo humano, Nueva York, PNUD. En Internet: http://hdr.undp.org/ en/media/HDR_2009_ES_Summary.pdf

RIST, G., (2002), El desarrollo: historia de una creencia occidental, Madrid, Catarata.

RUIZ BALZOLA, A., (2005), «Transnational migratory spaces: the emergent of a new context for public policies» en SETIÉN, M.L. y VICENTE, T. (ed.), Cross-disciplinary Views on migration Diversity, Bilbao, University of Deusto.

SANAHUJA, J.A., (2007), «Desigualdad, pobreza y desarrollo», en E. BARBÉ, Relaciones Internacionales, Madrid, Tecnos, pp. 344-361.
SEN, A., (1999), Development as Freedom, New York, Anchor Books.

SODUPE, K., (2003), La teoría de las Relaciones Internacionales a comienzos del siglo XXI, Bilbao, Universidad del País Vasco.

SOTILLO, J.A., (2011), El sistema de cooperación para el desarrollo: actores, formas y procesos, Madrid, Instituto Universitario de Desarrollo y Cooperación (IUDC) y Catarata.

SUTCLIFFE, B., (1995), «Desarrollo frente a economía», en Revista de Ecología Política, $n^{\circ}$ 9, pp. 27-49.

UNCETA, K., (2009), «Desarrollo, subdesarrollo, maldesarrollo y postdesarrollo», en Carta Latinoamericana. Contribuciones en desarrollo y sociedad en América Latina, $\mathrm{n}^{\circ} 7$, pp. 1-34. En Internet: http:// pdf2.hegoa.efaber.net/entry/content/455/ LatinoAmericana07Unceta09-1.pdf

UNIÓN EUROPEA, (2005), Conclusiones de la Presidencia del Consejo Europeo de Bruselas, 15-16 de diciembre de 2005, en Internet: http://www.maec.es/SiteCollectionDocuments/Espana \%20y\%20 la $\%$ 20Union $\% 20$ Europea/Politicas $\% 20$ Comunitarias/MedioAmbiente/87658.pdf WALLERSTEIN I., (1979), El moderno sistema mundial. La agricultura capitalista y los orígenes de la economía-mundo europea en el siglo XVI. Tomo I, Madrid, Siglo XXI.

ZAPATA-BARRERO, R. y ZARAGOZA CRISTIANI, J., (2008), «Externalización de las políticas de inmigración en España: ¿giro de orientación política en la gestión de fronteras y de flujos migratorios?", en Panorama Social, $n^{\circ}$ 8: Inmigrantes en España: Participación y convivencia, Madrid, Fundación de las Cajas de Ahorros (Funcas), pp. 186-195.

\section{REFERENCIAS ELECTRÓNICAS:}

\section{BANCO MUNDIAL:}

http://datos.bancomundial.org/income-level/ LIC [Actualizado en octubre de 2011]. 
NACIONES UNIDAS:

http:/www.un.org/milleniumgoals/pdf/ mdg2007.pdf [Actualizado en octubre de 2011].

UNIÓN EUROPEA:

http://europa.eu/rapid/press-release_MEMO11-221_en.htm?locale=en [Actualizado en abril de 2013].
PNUD:

http://hdr.undp.org/es/desarrollohumano/ [Actualizado en octubre de 2011].

CEPAL:

http://www.eclac.cl/cooperacion/ [Actualizado en octubre de 2011]. 